\title{
A Phase 1 Dose Escalation Study of Eribulin in Combination with Weekly Carboplatin for the Treatment of Metastatic Breast Cancer
}

\author{
Aixa Elena Soyano ${ }^{1, ~ *, ~ M i c h a e l ~ S h a f i q u e ~}{ }^{2}$, Roohi Ismail-Khan ${ }^{3}$, Dawn Goodridge ${ }^{4}$, David Boulware ${ }^{5}$, \\ Hatem Soliman ${ }^{1}$, Hyo Sook Han ${ }^{1}$ \\ ${ }^{1}$ Department of Breast Oncology, H. Lee Moffitt Cancer Center and Research Institute, Tampa, USA \\ ${ }^{2}$ Department of Thoracic Oncology, H. Lee Moffitt Cancer Center and Research Institute, Tampa, USA \\ ${ }^{3}$ Satellite and Community Oncology Program, H. Lee Moffitt Cancer Center and Research Institute, Tampa, USA \\ ${ }^{4}$ Department of Late Phase Research, Florida Cancer Specialists \& Research Institute, Tampa, USA \\ ${ }^{5}$ Department of Biostatistics, H. Lee Moffitt Cancer Center and Research Institute, Tampa, USA \\ Email address: \\ Aixa.soyano@moffitt.org (A. E. Soyano) \\ *Corresponding author
}

\section{To cite this article:}

Aixa Elena Soyano, Michael Shafique, Roohi Ismail-Khan, Dawn Goodridge, David Boulware, Hatem Soliman, Hyo Sook Han. A Phase 1 Dose Escalation Study of Eribulin in Combination with Weekly Carboplatin for the Treatment of Metastatic Breast Cancer. Cancer Research Journal. Vol. 9, No. 3, 2021, pp. 171-175. doi: 10.11648/j.crj.20210903.17

Received: August 2, 2021; Accepted: August 24, 2021; Published: August 31, 2021

\begin{abstract}
Background: Metastatic breast cancer is a common and devastating diagnosis. New strategies for treatment are needed to help improve outcomes. Eribulin is an anti-microtubule agent approved in 2010 for advanced breast cancer. Combination with other chemotherapeutic agents provides an alternative treatment option for these patients. Purpose: This study evaluates the safety, tolerability and activity of eribulin and weekly carboplatin in a dose-escalation schema in patients with metastatic breast cancer. Methods: Patients were treated with eribulin and carboplatin AUC 2 administered on the first and eighth days of a 21-day cycle. Three doses of eribulin $\left(0.9,1.1\right.$ and $\left.1.4 \mathrm{mg} / \mathrm{m}^{2}\right)$ were examined. An additional 10 patients were enrolled into an expansion cohort at the recommended Phase 2 dose. Results: A total of 19 patients were treated, including 10 patients in the dose expansion cohort. There was no dose limiting toxicity related to the study therapy in the dose escalation cohorts. Grade 3 toxicities included neutropenia $(21 \%)$, anemia $(10 \%)$, fatigue $(10 \%)$, peripheral sensory neuropathy (10\%), infusion related reactions $(5 \%)$, pericardial effusion $(5 \%)$, diarrhea $(5 \%)$ and pleural effusion $(5 \%)$. Twenty-six percent of patients had grade 4 neutropenia, but there were no events of sepsis or febrile neutropenia. The maximum tolerated dose (MTD) of eribulin in combination with carboplatin AUC 2 was determined to be $1.4 \mathrm{mg} / \mathrm{m}^{2}$. Four patients experienced clinical benefit, 2 patients with stable disease greater than 6 months and 2 patients with partial response, demonstrating a clinical benefit rate of $21 \%$. Conclusion: Eribulin and weekly carboplatin appeared to be safe and well tolerated with demonstrated clinical benefit. The recommended Phase 2 dose level was $1.4 \mathrm{mg} / \mathrm{m}^{2}$ of eribulin. Further studies can be pursued for this combination regimen to establish its efficacy.
\end{abstract}

Keywords: Eribulin, Breast Neoplasms, Drug Therapy, Breast Cancer

\section{Introduction}

Globally, breast cancer is the most commonly diagnosed malignancy after skin cancers. In the United States, it is the second most diagnosed cancer in women and the secondleading cause of cancer related death. [1] The incidence of new cases of invasive breast cancer is estimated to be over 280,000 in 2021, with over 43,000 deaths attributed to it [2]. Survival for invasive breast cancer has improved dramatically. Specifically, metastatic breast cancer has a median overall survival approaching two years, an improvement over the last 25 years likely related to improved hormonal, targeted and chemotherapeutic agents.[3] Anthracyclines and taxanes, have been associated with improved overall survival, and these 
agents are typically administered in the adjuvant setting. [4] However, in the setting of metastatic breast cancer the vast majority of patients are diagnosed in the recurrent setting, and a large number of patients have been exposed to these agents. $[5,6]$ It is imperative to develop new strategies to treat patients with such refractory disease.

In 2010, eribulin was approved to treat metastatic breast cancer in patients who received at least two prior chemotherapy regimens. [7] It is an analogue of halichondrin $\mathrm{B}$, a naturally occurring substance found in marine sponges. [6, 8] It acts on microtubules with a mechanism of action that is distinct from the taxanes. [9-12] In the pivotal Phase III trial which led to this approval, the patient population received both anthracycline and taxane prior to eribulin. [13] Compared to investigator's choice of chemotherapy, eribulin was associated with a nearly 3-month improvement in median overall survival. Therefore, eribulin is used particularly in the taxane resistant setting. [13]

A Phase 1 study evaluating eribulin (Day 1 and 8) in combination with carboplatin (day 1) on a 21-day schedule in advanced solid tumors was conducted in two stages. This study found the maximum tolerated dose of eribulin in combination with carboplatin AUC 5 to be $1.4 \mathrm{mg} / \mathrm{m}^{2}$ and $1.1 \mathrm{mg} / \mathrm{m}^{2}$ with carboplatin AUC 6 [14]. Across both stages, $40 \%$ of patients experienced grade 3 or 4 neutropenia, and $29 \%$ experienced Grade 3 or 4 thrombocytopenia. A retrospective study using eribulin $1.1 \mathrm{mg} / \mathrm{m}^{2}$ on day 1 and 8 with carboplatin AUC 5 on day 1 on a 21-day schedule at a single institution in patients with previously treated metastatic breast cancer demonstrated similar toxicity with a $20 \%$ rate of hospitalization for febrile neutropenia [15]. In the neoadjuvant setting, eribulin $1.4 \mathrm{mg} / \mathrm{m}^{2}$ was used in combination with carboplatin AUC 6 in patients with Stage IIII breast cancer. [16] Grade 3 to 4 neutropenia still occurred in nearly $60 \%$ of patients, with $19 \%$ experiencing grade 3 and 4 thrombocytopenia. Though the combination may be tolerable, the hematologic toxicity profile is severe. Carboplatin AUC 2 has been combined with paclitaxel and gemcitabine in the advanced breast cancer setting with good tolerability and improved hematologic toxicity $[17,18]$. The goal of this trial was to establish the safety, tolerability, and preliminary anti-tumor activity of combination Day 1 and 8 eribulin with carboplatin at AUC 2 on a 21-day schedule.

\section{Patients and Methods}

\subsection{Eligibility}

This Phase 1 trial enrolled patients with unresectable locally advanced or metastatic HER2 negative breast cancer. Patients must have received no more than 3 prior chemotherapeutic regimens in the setting of metastatic disease, and standard curative measures must have failed in patients with locally advanced or unresectable disease. Eligible patients must have been over 18 years of age at enrollment, had histologically or cytologically confirmed breast cancer, and had an Eastern Cooperative Oncology Group (ECOG) score of 0-2. Patients had normal organ and marrow function defined as: white blood cell count $\geq 3,000 / \mu \mathrm{L}$ with absolute neutrophil count $\geq 1,500 \mu \mathrm{L}$; platelet count $\geq 100,000 \mu \mathrm{L}$; total bilirubin within normal institutional limits; aspartate aminotransferase and alanine aminotransferase levels $\leq 2.5$ times the institutional upper limit of normal; creatinine within normal institutional limits or a creatinine clearance $\geq 60 \mathrm{~mL} / \mathrm{min} / 1.73 \mathrm{~m}^{2}$ for patients with creatinine above the institutional normal. Patients of childbearing potential must have had a negative serum B-hCG pregnancy test, and patients of childbearing age and their partners agreed to use adequate contraception during study participation. Ability to understand and willingness to sign informed consent was also a requisite.

Patients were excluded from participation if they received chemotherapy or radiotherapy within 2 weeks before enrolling in the study. Patients with adverse events or toxicities from prior agents administered must have recovered from these effects, and any residual toxicity cannot be more than grade 1 in severity. Patients participating on or those who had participated in a study of an investigational agent or device must have been off trial for at least 30 days prior to enrollment in this study. Patients with active brain metastatic disease or carcinomatous meningitis were excluded, except those with stable disease for at least 2 months prior to entry. Other exclusion criteria included: history of allergic reactions attributed to compounds of similar chemical or biological structure as the study agents; active Hepatitis A or a history of Hepatitis B, C or HIV; uncontrolled intercurrent illness; peripheral neuropathy of severity greater than grade 1 as a baseline; concurrent use of Class Ia and III antiarrhythmics. No patient received prior eribulin or platinum chemotherapy.

This trial was approved by the University of South Florida institutional review board (IRB) and conducted in keeping with all applicable institutional policies and federal regulations.

\subsection{Treatment}

Three dose levels for eribulin at $0.9,1.1$ and $1.4 \mathrm{mg} / \mathrm{m}^{2}$ in combination with carboplatin AUC 2 were evaluated in this phase 1 study. Cohorts of 3 patients were enrolled on each escalating dose of eribulin administered IV immediately prior to carboplatin. Both medications were given on days 1 and 8 of a 21-day cycle. Dose-limiting toxicities (DLT) were assessed during cycle 1 of therapy. Once the maximum tolerated dose was reached, an additional 10 patients were treated at this dose for dose expansion cohort. There was no pre-planned number of treatment cycles, and patients were treated until progression, unacceptable toxicity, or withdrawal of consent. Patients were taken off the study treatment if treatments were delayed greater than 3 weeks or if they required more than 2 dose reductions.

A standard $3+3$ trial design was employed. Toxicity severity was graded according to guidelines outlined in the NCI-CTCAE Version 4.0. Dose limiting toxicity (DLT) was defined as adverse events related to study treatment defined as following: Hematologic dose-limiting toxicity including grade 4 neutropenia lasting for $\geq 7$ days; grade 3 or 4 
neutropenia with fever $\geq 38.5^{\circ} \mathrm{C}$ and/or infection requiring antibiotic or anti-fungal treatment; or grade 4 thrombocytopenia. Non-hematologic dose-limiting toxicity was defined as any grade 3, 4 or 5 with the exception of: grade 3 nausea, vomiting, diarrhea, dehydration or hyperglycemia in the setting of inadequate compliance with supportive measures; grade 3 or higher potassium or magnesium abnormalities in the setting of inadequate compliance with supportive measures; alopecia; or inadequately treated hypersensitivity reactions.

Dose delays and modifications were allowed, with all dose reductions being permanent for an individual patient. Supportive care guidelines allowed G-CSF (Neulasta or Neupogen) to be used for cycle 2 and beyond in the dose escalation phase at the discretion of the treating physician. All patients received growth factor support with cycle 1 in the expansion cohort. Toxicity was monitored with history, physical exam, CBC and serum chemistries on days 1 and 8 of each 21-day cycle. Adverse event evaluation was performed throughout the study.

Restaging radiologic scans and tumor measurements were performed in the first 6 weeks from the start of therapy, thereafter, every 9 weeks.

\subsection{Endpoints/Statistics}

This trial's primary objective was to identify the maximum tolerated dose (MTD) of eribulin in combination with carboplatin dosed for an AUC of 2. The MTD was defined as the dose of eribulin at which the percentage of patients experiencing a DLT is closest to $30 \%$. Secondary objectives included safety, tolerability, and activity of the combination of eribulin and carboplatin. To evaluate activity, response rate was calculated for subjects in the study overall. Response and progression were defined using Response and Evaluation Criteria in Solid Tumors (RECIST) Version 1.1. [19] Confirmation of partial response (PR) and complete response (CR) was performed no less than 4 weeks after criteria for response were first met. To qualify as stable disease (SD), tumor measures must have met SD criteria at least once at a minimum of 6 weeks from entry to study.

\section{Results}

From Feb 2013 to Nov 2014, 19 patients were enrolled at Moffitt Cancer Center. Patient characteristics are summarized in Table 1. All patients were female, and the median age was 55 years. Eight patients (42\%) had triple-negative breast cancer and 18 patients had visceral disease. Seventeen out of 19 patients received prior both taxanes and anthracyclines, including 11 patients in the adjuvant or neoadjuvant setting. The median number of prior chemotherapies in the setting of metastatic disease was 1.6 (range 0-3).

The combination therapy was generally well tolerated. Table 2 summarizes the observed grade 3 and 4 adverse events. The only grade 4 toxicity seen was neutropenia, which $26 \%$ of patients experienced. Twenty-one percent of patients experienced grade 3 neutropenia. The incidence of significant neutropenia was less in the dose expansion cohort, likely due to the use of growth factor support. Only 1 patient experienced grade 3 neutropenia and 2 patients had grade 4 neutropenia in the expansion cohort. There were no hospitalizations, however, for sepsis, fever or febrile neutropenia. Other grade 3 toxicities included anemia (10\%), pericardial effusion $(10 \%)$, diarrhea $(10 \%)$, fatigue $(10 \%)$, peripheral sensory neuropathy $(10 \%)$, infusion related reactions $(5 \%)$, and pleural effusion $(5 \%)$. There were no events of grade 3 or grade 4 thrombocytopenia.

Table 1. Baseline Data in Study Population ( $n=19)$.

\begin{tabular}{ll}
\hline Demographics and Clinical Characteristics & No. (\%) \\
\hline Age years & \\
Median & 55.0 \\
Range & $35-73$ \\
Race & \\
White & $14(73.7)$ \\
Black & $4(21.0)$ \\
Asian & $1(5.3)$ \\
Ethnicity & \\
Hispanic or Latino & $1(5.3)$ \\
Non-Hispanic & $18(94.7)$ \\
ECOG performance status (at baseline) & \\
0 & $10(52.6)$ \\
1 & $9(47.4)$ \\
Visceral Disease & \\
Yes & $18(94.7)$ \\
No & $1(5.3)$ \\
Sites of Metastatic Disease & \\
1 & $2(10.5)$ \\
2 & $6(31.6)$ \\
3 & $9(47.4)$ \\
$>3$ & $2(10.5)$ \\
Prior Therapy for Metastatic Disease & \\
None & $1(5.3)$ \\
Chemotherapy & $16(84.2)$ \\
Hormonal Therapy (If ER/PR positive) & $10(52.6)$ \\
\hline
\end{tabular}

Table 2. Summary of Grade 3 and 4 Adverse Events: Total Subjects $(n=19)$.

\begin{tabular}{lllll}
\hline \multirow{2}{*}{ AE Description } & \multicolumn{2}{l}{ Grade 3 } & \multicolumn{2}{l}{ Grade 4 } \\
\cline { 2 - 6 } & $\mathbf{n}$ & $\mathbf{\%}$ & $\mathbf{n}$ & $\mathbf{\%}$ \\
\hline Anemia & 2 & 10.53 & 0 & 0.00 \\
Pericardial effusion & 1 & 5.26 & 0 & 0.00 \\
Eye pain & 1 & 5.26 & 0 & 0.00 \\
Diarrhea & 1 & 5.26 & 0 & 0.00 \\
Fatigue & 2 & 10.53 & 0 & 0.00 \\
Infusion related reaction & 1 & 5.26 & 0 & 0.00 \\
Lymphocyte count decreased & 1 & 5.26 & 0 & 0.00 \\
Neutrophil count decreased & 4 & 21.05 & 5 & 26.32 \\
White blood cell decreased & 2 & 10.53 & 0 & 0.00 \\
Hypokalemia & 1 & 5.26 & 0 & 0.00 \\
Hyponatremia & 1 & 5.26 & 0 & 0.00 \\
Hypophosphatemia & 0 & 0.00 & 1 & 5.26 \\
Back pain & 1 & 5.26 & 0 & 0.00 \\
Myalgia & 1 & 5.26 & 0 & 0.00 \\
Peripheral sensory neuropathy & 2 & 10.53 & 0 & 0.00 \\
Dyspnea & 1 & 5.26 & 0 & 0.00 \\
Pleural effusion & 1 & 5.26 & 0 & 0.00 \\
Respiratory, thoracic and mediastinal & 1 & 5.26 & 0 & 0.00 \\
disorders - Other, specify & & & & \\
Skin and subcutaneous tissue disorders - & 1 & 5.26 & 0 & 0.00 \\
Other, specify & 1 & 5.26 & 0 & 0.00 \\
Thromboembolic event & & & &
\end{tabular}


The maximum tolerated dose of eribulin in combination with carboplatin AUC 2 was identified as $1.4 \mathrm{mg} / \mathrm{m}^{2}$. Two patients came off the trial therapy due to grade 3 carboplatin infusion reaction (escalation cohort) and grade 4 neutropenia (expansion cohort). Four patients experienced clinical benefit, 2 with SD greater than 6 months and 2 with PR, demonstrating a clinical benefit rate of $21 \%$.

Median progression free survival (PFS) was estimated to be 2.3 months (95\% CI 1.35, 3.22 months), and median overall survival was 13.6 months (95\% CI 4.73, 18.67 months). In the triple-negative population, the median PFS was 2.4 months (95\% CI 0.76, 3.22 months). The overall survival in this population was 7.6 months $(95 \%$ CI 2.40 , 18.67 months).

Patients were on treatment for an average of 3 months. The longest duration of treatment on study was nearly 8 months. This was observed in a patient with triple-negative disease. The patient completed 10 cycles of carboplatin and eribulin with delays following cycles 2 and 9 for neutropenia.

\section{Discussion}

The FDA approved eribulin to treat metastatic breast cancer in 2010 based on the results of the EMBRACE trial. [18] At that time, it was the first trial to show an improved OS in this heavily pre-treated population of breast cancer patients. The authors demonstrated that the toxicities associated with treatment were manageable. Likewise, the results of this Phase 1 trial indicate that the combination of eribulin and carboplatin in a heavily pretreated population is safe and tolerable with clinically relevant response rates. Our study resulted in no treatment-related deaths.

Previous trials using combination of eribulin with higher doses of carboplatin (AUC 5-6) have demonstrated a significant incidence of severe hematologic toxicities. [14, $15,16]$ However, in our trial no hematologic DLTs were experienced. The most common hematologic adverse event, however, was grade 1 neutropenia, experienced by $32 \%$ of patients. Severe, grade 3 or 4 neutropenia occurred in $21 \%$ and $26 \%$ of patients, respectively. However, these events led to the discontinuation of therapy for only 1 patient, and there were no admissions for sepsis, infection, or febrile neutropenia. The prophylactic use of GCSF should be considered in future studies with this regimen. As opposed to previous studies, which reported that $>20 \%$ of patients who received combinations with higher doses of carboplatin developed Grade 3 or 4 thrombocytopenia, the severity of thrombocytopenia in this regimen was low with Grade 1 $(16 \%)$ and Grade $2(11 \%)[14,15,16]$.

Interestingly, a patient with the longest time on treatment had triple negative breast cancer. She received a total of 10 cycles of chemotherapy with only 2 interruptions for neutropenia. Her best response was stable disease, but ultimately progressed on treatment. Additionally, one of the only 2 patients who demonstrated a partial response had triple-negative disease that was associated with a BRCA1 mutation. This patient ultimately progressed after 6 cycles of therapy. As a population, the patients with triple-negative breast cancer received prior therapy including taxanes and anthracyclines during the course of their treatment except one patient, who presented with de novo metastatic disease. The median PFS was estimated to be 2.4 months with a median OS of 7.58 months. Furthermore, several patients who experienced clinically meaningful benefit from this regimen had triple-negative disease, one of whom had a BRCA1 mutation.

\section{Conclusion}

Eribulin in combination with carboplatin AUC 2 was well tolerated in patients with pretreated advanced metastatic breast cancer with a meaningful clinical benefit rate at $21 \%$. The toxicity profile was favorable with expected limited and low-grade adverse events. This treatment combination is a reasonable option for patients in the taxane refractory setting. Further investigation of this approach can be further explored for triple negative breast cancer given limited options available in this population.

\section{Compliance with Ethical Standards}

\section{Ethical Approval}

This trial was approved by the University of South Florida institutional review board (IRB) and conducted in keeping with all applicable institutional policies and federal regulations. All procedures performed in studies involving human participants were in accordance with the ethical standards of the institutional and/or national research committee and with the 1964 Helsinki declaration and its later amendments or comparable ethical standards.

\section{Informed Consent}

Informed consent was obtained from all individual participants included in the study.

\section{Conflict of Interest}

The authors declare that they have no competing interests.

\section{References}

[1] Siegel RL, Miller KD, Fuchs HE, Jemal A: Cancer Statistics, 2021. CA Cancer J Clin 2021, 71(1):7-33.

[2] Cancer Stat Facts: Female Breast Cancer [https://seer.cancer.gov/statfacts/html/breast.html]

[3] Chia SK, Speers CH, D'Yachkova Y, Kang A, Malfair-Taylor S, Barnett J, Coldman A, Gelmon KA, O'Reilly S E, Olivotto IA: The impact of new chemotherapeutic and hormone agents on survival in a population-based cohort of women with metastatic breast cancer. Cancer 2007, 110 (5): 973-979.

[4] O'Shaughnessy J: Extending survival with chemotherapy in metastatic breast cancer. Oncologist 2005, 10 Suppl 3: 20-29. 
[5] Zeichner SB, Herna S, Mani A, Ambros T, Montero AJ, Mahtani RL, Ahn ER, Vogel CL: Survival of patients with denovo metastatic breast cancer: analysis of data from a large breast cancer-specific private practice, a university-based cancer center and review of the literature. Breast Cancer Res Treat 2015, 153 (3): 617-624.

[6] Moreno-Aspitia A, Perez EA: Treatment options for breast cancer resistant to anthracycline and taxane. Mayo Clin Proc 2009, 84 (6): 533-545.

[7] Halaven (eribulin) [prescribing information]. Woodcliff Lake, NJ: Eisai Inc; February 2021

[8] Bai RL, Paull KD, Herald CL, Malspeis L, Pettit GR, Hamel E: Halichondrin $\mathrm{B}$ and homohalichondrin $\mathrm{B}$, marine natural products binding in the vinca domain of tubulin. Discovery of tubulin-based mechanism of action by analysis of differential cytotoxicity data. J Biol Chem 1991, 266 (24): 15882-15889.

[9] Hirata Y, Uemura D: Halichondrins-antitumor polyether macrolides from a marine sponge. Pure and Applied Chemistry 1986, 58 (5): 701-710.

[10] Kuznetsov G, Towle MJ, Cheng H, Kawamura T, TenDyke K, Liu D, Kishi Y, Yu MJ, Littlefield BA: Induction of morphological and biochemical apoptosis following prolonged mitotic blockage by halichondrin B macrocyclic ketone analog E7389. Cancer Res 2004, 64 (16): 5760-5766.

[11] Okouneva T, Azarenko O, Wilson L, Littlefield BA, Jordan MA: Inhibition of centromere dynamics by eribulin (E7389) during mitotic metaphase. Mol Cancer Ther 2008, 7 (7): 20032011.

[12] Smith JA, Wilson L, Azarenko O, Zhu X, Lewis BM, Littlefield BA, Jordan MA: Eribulin binds at microtubule ends to a single site on tubulin to suppress dynamic instability. Biochemistry 2010, 49 (6): 1331-1337.

[13] Twelves C, Cortes J, Vahdat LT, Wanders J, Akerele C,
Kaufman PA: Phase III trials of eribulin mesylate (E7389) in extensively pretreated patients with locally recurrent or metastatic breast cancer. Clin Breast Cancer 2010, 10 (2): 160-163.

[14] Swami U, Petrylak DP, Raftopoulos H, Shuster DE, Wang G, Kumar V, Martinez G, Goel S, Aisner J: Phase IB study of eribulin mesylate in combination with carboplatin in patients with advanced solid tumors. J Clin Oncol 2010, 28 (15): 2589.

[15] Romano PM, Baraibar I, Fernandez-Hidalgo O, Santisteban M, Espinos J, Salas D, Sala P, Gardeazabal I, Oteiza LZ, Aramendia JM: Eribulin and carboplatin in heavily pretreated patients with metastatic breast cancer: A single institution experience. J Clin Oncol 2015, 33 (28): 122.

[16] Kaklamani VG, Jeruss JS, Hughes E, Siziopikou K, Timms KM, Gutin A, Abkevich V, Sangale Z, Solimeno C, Brown KL et al: Phase II neoadjuvant clinical trial of carboplatin and eribulin in women with triple negative early-stage breast cancer (NCT01372579). Breast Cancer Res Treat 2015, 151 (3): 629-638

[17] Loesch D, Robert N, Asmar L, Gregurich MA, O'Rourke M, Dakhil S, Cox E: Phase II multicenter trial of a weekly paclitaxel and carboplatin regimen in patients with advanced breast cancer. J Clin Oncol 2002, 20 (18): 3857-3864.

[18] Cortes J, O'Shaughnessy J, Loesch D, Blum JL, Vahdat LT, Petrakova K, Chollet P, Manikas A, Diéras V, Delozier T et al: Eribulin monotherapy versus treatment of physician's choice in patients with metastatic breast cancer (EMBRACE): a phase 3 open-label randomised study. Lancet 2011, 377 (9769): 914-923.

[19] Eisenhauer EA, Therasse P, Bogaerts J, Schwartz LH, Sargent D, Ford R, Dancey J, Arbuck S, Gwyther S, Mooney M et al: New response evaluation criteria in solid tumours: revised RECIST guideline (version 1.1). Eur J Cancer 2009, 45 (2): 228-247. 